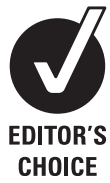

${ }^{1}$ British Medical Association, Ethics Department, BMA House, London, UK

${ }^{2}$ Médecins Sans Frontières, London, UK

\section{Correspondence to} Dr Tejshri Shah, Médecins Sans Frontières, 67-74 Saffron Hill, London, EC1N 80X, UK; tejshrishah@gmail.com

Received 9 August 2010 Accepted 7 October 2010 Published Online First 17 November 2010

\title{
Ethical dilemmas in medical humanitarian practice: cases for reflection from Médecins Sans Frontières
}

\author{
Julian Sheather, ${ }^{1}$ Tejshri Shah ${ }^{2}$
}

\begin{abstract}
Médecins Sans Frontières (MSF) is an independent medical humanitarian organisation working in over 70 countries. It has provided medical assistance for over 35 years to populations vulnerable through conflict, disease and inadequate health systems. Medical ethics define the starting point of the relationship between medical staff and patients. The ethics of humanitarian interventions and of research in conflict settings are much debated. However, less is known about the ethical dilemmas faced by medical humanitarian staff in their daily work. Ethical dilemmas can be intensified in humanitarian contexts by insecure environments, lack of optimum care, language barriers, potentially heightened power discrepancies between care providers and patients, differing cultural values and perceptions of patients, communities and medical staff. Time constraints, stressful conditions and lack of familiarity with ethical frameworks can prevent reflection on these dilemmas, as can frustration that such reflection does not necessarily provide instant solutions. Lack of reflection, however, can be distressing for medical practitioners and can reduce the quality of care. Ethical reflection has a central role in MSF, and the organisation uses ethical frameworks to help with clinical and programmatic decisions as well as in deliberations over operational research. We illustrate and discuss some real ethical dilemmas facing MSF teams. Only by sharing and seeking guidance can MSF and similar actors make more thoughtful and appropriate decisions. Our aim in sharing these cases is to invite discussion and dialogue in the wider medical community working in crisis, conflict or with severe resource limitations.
\end{abstract}

\section{INTRODUCTION}

Médecins Sans Frontières (MSF) is an independent medical humanitarian organisation working in over 70 countries. It has provided medical assistance for over 35 years to populations vulnerable through conflict, disease and inadequate health systems. Medical ethics define the starting point of the relationship between medical staff and patients. The organisation's charter and guiding documents state that 'MSF volunteers undertake to respect their professional code of ethics' ${ }^{1}$ and 'MSF missions are carried out in the respect of the rules of medical ethics, in particular the duty to provide care without causing harm to either individuals or groups. Each person in danger will be assisted with humanity, impartiality and in respect of medical confidentiality. ${ }^{2}$ Despite the clarity of this vision and of many other guiding policies, the translation of ethical principles and policies into the reality of humanitarian assistance can be messy and complex.
The ethics of humanitarian interventions and of research in conflict settings ${ }^{3-6}$ are much debated. However, less is known about the ethical dilemmas faced by medical humanitarian staff in their daily work. ${ }^{7} 8$ Ethical dilemmas can be intensified in humanitarian contexts by insecure environments, lack of optimum care, language barriers, potentially heightened power discrepancies between care providers and patients, differing cultural values and perceptions of patients, communities and medical staff. Time constraints, stressful conditions and lack of familiarity with ethical frameworks can prevent reflection on these dilemmas, as can frustration that such reflection does not necessarily provide instant solutions. Lack of reflection, however, can be distressing for medical practitioners ${ }^{7} 8$ and can reduce the quality of care.

Ethical reflection has a central role in MSF, and the organisation uses ethical frameworks to help with clinical and programmatic decisions as well as in deliberations over operational research. This desire for reflection has led to this paper; however, we do not wish to debate ethical frameworks here. Instead, we illustrate and discuss, with the guidance of a professional ethicist, some real ethical dilemmas facing MSF teams. Only by sharing and seeking guidance can MSF and similar actors make more thoughtful and appropriate decisions. Our aim in sharing these cases is to invite discussion and dialogue in the wider medical community working in crisis, conflict or with severe resource limitations. For security and confidentiality some details have been omitted.

\section{HIV TESTING}

In many locations, safe blood transfusions must be delivered without the luxury of a pre-existing screened blood bank. Potential donors are often the patient's friends or relatives but can include individuals from the local community who volunteer to donate. MSF laboratory staff screen blood for a range of factors, including HIV, to see whether it can be used. A health worker counsels donors before testing. If a donor is tested positive for a transmissible infection, such as HIV, they are told that their blood is not compatible and are asked if they want to know the reason for this.

\section{MSF dilemma 1}

The stigma around HIV can be high. A diagnosis can lead to rejection by family and community, and in some instances, a person suspected to be HIVpositive may even be killed. Where MSF is not running a programme offering antiretroviral therapy or where referral is impossible, our laboratory staff, despite a clear policy, can fear informing 
or counselling individuals who test positive for HIV. In these contexts, our staff struggle with knowing the results and yet not informing the individual. Is there any way to reconcile this tension?

\section{Ethicist's response}

There is a presumption in contemporary western medical ethics that patients should be fully informed, that information should not be withheld, even if its knowledge would not be in a patient's best interests. This is a medical expression of a high principle in western culture-that of respecting the right of individuals to make informed decisions, the right to self-rule or autonomy as it is sometimes known.

Blood for transfusion must of course be screened. If a condition, such as HIV, is identified then, given the principle of respect for autonomy, the donor should be informed. This information has a number of obvious benefits. It enables the donor to seek treatment, either to cure or mitigate symptoms. It enables them to plan for the future. Crucially, it enables them to minimise the risk to others, to practise safe sex, to avoid activities that might lead to transmission.

What happens, however, if the knowledge itself could be harmful? If there is no treatment available, no possibility of cure or mitigation of symptoms, then the benefits of disclosure diminish-although clearly, given the opportunity to minimise risks of transmission, they do not disappear-and the harms weigh more heavily.

Several practical considerations come to mind. Is there a way of minimising the harms involved? Can the information be given in a way that avoids suspicion? The right of the individual to make informed choices is at stake here; why can he not be given the information in absolute confidence? Where no treatment or referral is available, could potential donors be asked in advance whether they would like to receive the results of screening for any particular condition? In this way, the problems that disclosure could present can be discussed and the individual's freedom to make decisions be respected.

If there are few benefits that would stem from disclosure, and a host of possible dangers that the individual could not control, then it might be appropriate to consider withholding information. Given that disclosure in this case could help an individual prevent onward transmission, the risks of harm would have to be very significant indeed. Whenever professionals are working in cultures with which they are unfamiliar, great care must be taken in distinguishing cultural assumptions from reality. Discussing the issues with people with greater experience of the host culture is always worthwhile.

\section{What happened in the MSF programme?}

In one location where this issue has arisen, there is no acknowledgement by the community or even by many health staff that HIV is present in the population. So far, the MSF team have concluded that the potential harms in informing donors outweigh the benefits. The laboratory team give feedback that the blood is not compatible but do not feel comfortable counselling on the reasons why this is so. The field team initiated a broader discussion, in order to share the dilemma and recognising that the practice was not ideal. The plan now is to start sharing the information on the number of positive HIV test results with the community in the hope of dispelling the myth that HIV does not exist. How to share this information will be carefully considered and tactfully undertaken, with advice from key community members, as even this process will not be free of risks.

\section{FEMALE GENITAL MUTILATION}

MSF works in a variety of cultures and local values can clash with those of the organisation. For example, MSF has formulated a policy against female genital mutilation (FGM). ${ }^{9}$ FGM is typically performed without true consent, often on young girls. The most severe form of FGM is type III-excision of part or all of the external genitalia and narrowing of the vulval orifice. This is known as infibulation. The health complications alone constitute violations of health rights. The rights of the child and women's rights are protected by several universal (and regional) instruments including the Universal Declaration of Human Rights, the UN Convention on the Elimination of All Forms of Discrimination against Women, and the African Charter on Human and People's Rights. The Maputo Protocol to the African Charter on Human and People's Rights expressly prohibits all forms of FGM. The World Medical Association is explicitly opposed to medical involvement in the practice. In many countries it is illegal and a doctor involved in such practices could be prosecuted.

\section{MSF dilemma 2: sterile equipment}

MSF teams have faced situations where a nurse who is part of the community, who understands the importance of sterile procedures, asks to use MSF's sterile equipment to perform FGM. MSF is often the only source of sterile equipment in the area and the team has felt that the practice in the community will not change in the short term. Should they turn a blind eye to the use of MSF equipment?

\section{MSF dilemma 3: re-infibulation}

To allow childbirth, it is necessary to surgically open an infibulation. After delivery, women (and their husbands) ask for restoration of the infibulation (re-infibulation), which involves re-suturing. MSF opposes re-infibulation and works to ensure that it is not undertaken in its delivery facilities. ${ }^{9}$ Although MSF opposes this practice, not performing re-infibulations risks jeopardising community trust, which could lead to women no longer attending the only safe childbirth service in the region. Should the staff break MSF's policy, honour the request of the mother, and avoid the risk of losing community acceptance?

\section{Ethicist's response}

There are many ethical issues surrounding FGM. It is strongly linked to cultural belonging and definitions of womanhood: a girl will not usually be considered a woman unless she has been 'cut'. Women who have not been circumcised find difficulty marrying.

Although there are other cultural practices that destroy healthy tissue-ear piercing, ritual male circumcision-these are not intended to inhibit ordinary human functioning and their harms are not as extreme as those of the severe forms of FGM. The destruction of healthy human tissue for its own sake runs counter to deep intuitions about the purposes of medicine.

Although there are two separate dilemmas here, in a sense they are different dimensions of a single problem. Given the near-unanimous international opposition to FGM, are there any circumstances in which it might be ethically justifiable for MSF or its individual practitioners to facilitate it? The first dilemma asks this question in relation to an individual patient: should the prohibition be set aside because this woman will benefit from the use of sterile resources? The second asks it in relation to the community: should MSF mute its opposition to re-infibulation to retain the trust of the community and therefore be able to deliver health services? 


\section{Considerations for protecting individuals}

Arguing from basic principles, the case against FGM is strong and participation could rapidly come to look like condoning and even supporting it. From this perspective, MSF should prohibit the use of resources for such practices and honour the policy that opposes re-infibulation. Against this principle-based approach, however, need to be set some arguments of a more consequentialist character. What about the consequences of these decisions? The lives of both the individual woman and of those others in the community could well be better, perhaps immeasurably so, if sterile equipment were used, and experienced medical professionals undertook some interventions.

All other things being equal, a lesser harm is to be preferred to a greater one. The difficulty is that all other things are not equal, and the calculus of benefit and harm here is complex. Overriding important principles can have significant consequences, and the requirement to put the patient's interests first has been raised to a principle in medical ethics. As in many instances where deep principles or interests clash, compromises have been reached. In relation to individual patients, health professionals who have felt compelled to act have found ways of minimally intervening-just enough to meet cultural demands while leaving tissue as intact as possible. They have also worked with communities with a view to educating them to the harms of the practice and to its eradication. However, health professionals and organisations such as MSF, have every right to say that they will have nothing to do with FGM. Participation, they argue, will surely prolong the practice.

\section{MSF and community confidence}

The ability of MSF to function in any cultural context will depend on trust and community engagement. An uncompromising set of western autonomy-based values could rapidly lead to rejection by the host community. There could not conceivably be a one-size-fits-all approach to this issue; rather it is a case for dialogue.

One approach is to recognise that while cultural practices may vary, they can be slightly different paths to the same goal. Cultures can express commitment to similar underlying values in various ways. Take the issue of autonomy. In liberal democracies, there is a strong expectation that competent individuals will make their own healthcare choices, supported by information and advice from health professionals. Countries with different political traditions might not put such emphasis on individual choice. In some cultures, for example, families will participate more in decisions. Although family participation can create difficulties around consent and confidentiality, both approaches contain important insights about human thriving.

Additionally, cultures are not monolithic but often dynamic and subject to internal critique and change. Productive input from other cultures, scientific and practical as well as ethical and value-based, can be an important driver of change. MSF could work alongside groups and individuals looking to change harmful cultural practices, without necessarily being vulnerable to charges of cultural relativism.

The problem with harmful traditional practices such as FGM is that, there may be wide consensus that it is unacceptable in any form. Furthermore, the act itself seems to undermine the goals that MSF strives to promote, and working with it or alongside it risks compromising the ethical integrity of the organisation. These circumstances call for difficult political and ethical judgement. There may be some justification for working with cultures with the longer-term aim of educating people to the harms of these practices with the intention, ultimately, of eliminating them. There will always be an attendant risk that the organisation is accused of complicity, of violating the interests of the individuals it is committed to benefiting. Wherever principles are invoked there will always be some need to negotiate. But there may be situations where the cost of such negotiation is too high and MSF should refuse to participate, even perhaps at the cost of being able to remain effective in the country.

\section{What happened in the MSF programme? Sterile equipment}

In one location the team decided not to give the nurse the sterile equipment to conduct infibulations and explained why. The nurse tried to dissuade parents from the practice by giving them an overview of the harms. However, she did conduct infibulations, if still requested, as for her it was too hard to refuse. The decision was an uncomfortable one, since the team was not supporting the nurse with sterile equipment. This discomfort meant that help was sought and a local non-governmental organisation was contacted. The non-governmental organisation held workshops on how FGM was not a useful practice. Later that year a senior male elder announced on the birth of his daughter that, unlike her older sisters, she would not undergo FGM. These statements were clearly far more acceptable than if they had been made by an international organisation. We do not know the extent of the workshops' influence but one girl at least was spared the procedure.

\section{Re-infibulation}

Re-infibulation was (and is) a difficult dilemma and opinions in MSF have differed. To simplify the debate, those closer to the projects often feared the backlash of the community, and those further away took a more zero tolerance approach. The tension encouraged one team to discuss the dilemma with men and women in the community. As a result they introduced the policy of non-re-infibulation. Re-infibulation is not openly conducted in MSF facilities but some staff are pressured to oblige the request and may do so covertly.

\section{ACTING BEYOND COMPETENCE}

MSF aims to deliver high quality healthcare with professionals suited to their tasks. Doctors and nurses will inevitably be asked to act above their competence level as resources, including specialist physicians, are limited. The following scenario and its variations are not uncommon.

\section{MSF dilemma 4}

Our doctor has previously assisted in caesarean sections but has never taken sole responsibility for one. The doctor who is responsible for surgery is on holiday and transport to the next surgical facility takes $7 \mathrm{~h}$, which is too long for this mother who is clearly in obstructed labour. The doctor is confronted with doing something that she has never done before. She knows that she could do a lot of harm by doing the operation badly, but doing nothing guarantees that the baby will not survive and may result in dangerous complications for the mother. The staff are waiting for her plan of action. What should she do?

\section{Ethicist's response}

Throughout their medical education, doctors are instructed to recognise and work within their competencies. This is to an extent predicated on the assumption that patients can be referred to a suitably qualified colleague. Even in resource-rich countries, however, emergencies arise where sufficient time is not available for referral. The regulatory body for doctors in the 
UK, the General Medical Council, states: 'in an emergency ... you must offer assistance, taking account of your own safety, your competence and the availability of other options for care'. ${ }^{10}$ In resource-poor countries, where specialist referral is far less available, doctors will often develop the ability to manage or treat a wide range of conditions that would be the preserve of specialists in more developed health systems. In this dilemma, however, the doctor is presented with an intervention that she knows to be outside her proven competence. The question she would have to ask is whether or not she had a reasonable belief that the intervention would be of overall benefit to the patient in comparison with other available options. Having medical training, and having assisted in caesarean sections, she clearly has some knowledge. An intervention in this context would clearly be risky, and she would have to weigh, to the best of her abilities, the risks of intervening or not.

\section{What happened in the MSF programme?}

In one instance, the doctor decided against intervening and chose to transfer the woman, in the hope of saving her if not her unborn child. However, the quality of the receiving facility was not clear. The mother was operated on but died (as well as her unborn child), with no further feedback on the reason why. Whether the outcome would have been different had the woman not been transferred is impossible to know. This case painfully demonstrates the reality of healthcare for many people and the pressures on health staff.

\section{CONCLUSIONS}

MSF has produced an ethics framework for operational research, ${ }^{11}$ ethics case studies for field staff, and training modules for managers and medics on medical ethics. The organisational culture encourages staff to share ethics problems with colleagues and seniors, but needs constant reinforcement to demonstrate that sharing difficulties is a strength and will receive an empathic response. Even reasonable people will disagree about many of these issues; managing different expectations requires skill and understanding, often in very tense situations. Engaging communities to help with dilemmas, especially those that occur frequently, can be useful in dispelling myths and assumptions. This approach has a long history in $\mathrm{MSF}^{12}$ and has been successful especially on issues as difficult to address as FGM but could certainly be further developed.

In the introduction, we stated that MSF missions respect the rules of medical ethics. The dilemmas presented highlight the frightening ease with which circumstances outpace even the most nuanced and carefully thought-through rules. They also demonstrate that although the past may be the best guide we have to the future, it is seldom good enough and new responses are always needed. MSF missions, by their nature, put health professionals in ethically challenging situations. Resource shortages, different cultural values, and the threat of social and political instability present enormous challenges. Morally, a dilemma arises when each available choice involves moral wrongdoing, that none is free from reproach. All the scenarios discussed here are dilemmas in this sense. MSF seeks to bring health goods to communities, but in doing so is forced to navigate harms. One interesting feature of these dilemmas is how they emerge from the impact of scientific knowledge on traditional cultures. Science identifies the HIV virus, calibrates the harms from FGM and creates the gold standard against which lesser health interventions are judged. And yet, as these scenarios show, this knowledge is not always seen as an unambiguous good. Though what this does suggest is that these dilemmas may be subject to historical change: as cultures move and adapt, so some of these tensions will disperse. This is a process to which MSF contributes. The dilemmas around HIV and FGM present a choice of ills. But by working with communities, by raising awareness of the benefits that medicine can bring, so attitudes change, and what seems like an intractable dilemma today is forgotten tomorrow.

Acknowledgements We thank Leslie Shanks and Michiel Lekkerkerker for comments on an earlier draft of this manuscript and Sarah Venis for editing assistance. We thank also the teams who inspired this article.

Competing interests None.

Contributors JS and TS wrote the paper and approved the final version of the manuscript. The corresponding author had full access to all the data in the study and had final responsibility for the decision to submit for publication.

Provenance and peer review Not commissioned; externally peer reviewed.

\section{REFERENCES}

1. Médecins Sans Frontières. The Charter of Médecins Sans Frontières. http://www. msf.org.uk/about_charter.aspx (accessed 12 Jan 2010).

2. Médecins Sans Frontières. Chantilly document. 1997. [Available upon request from Doctors Without Borders/Médecins Sans Frontières, 333 Seventh Avenue, 2nd floor, New York, NY 10001, USA.].

3. Ford N, Mills EJ, Zachariah $\mathrm{R}$, et al. Ethics of conducting research in conflict settings. Conflict Health 2009;3:7.

4. Schopper D. Research ethics review in humanitarian contexts: the experience of the independent ethics review board of Médecins Sans Frontières. Plos Med 2009:6:e1000115.

5. Sumathipala A, Siribaddana $S$. Research and clinical ethics after the tsunami: Sri Lanka. Lancet 2005;366:1418-20.

6. Leaning J. Ethics of research in refugee populations. Lancet 2001;357:1432-3.

7. Hunt MR. Ethics beyond borders: how health professionals experience ethics in humanitarian assistance and development work. Dev World Bioeth 2008; 8:59-69

8. Hunt MR. Resources and constraints for addressing ethical issues in medical humanitarian work: experiences of expatriate healthcare professionals. Am J Disaster Med 2009; 4:261-71

9. MSF Operations Centre Amsterdam. Position on Female Genital Mutilation. Amsterdam: MSF, 2006

10. General Medical Council. Good Medical Practice. London: General Medical Council, 2006.

11. MSF Ethics Review Board. Ethics framework for medical research. http:// fieldresearch.msf.org/msf/handle/10144/12364 (accessed 3 Nov 2009).

12. Ford N, Bedell R, eds. Intentions and Consequences: Human Rights, Humanitarianism and Culture. Amsterdam: MSF, 1999 